\title{
Minkowskian Solution of General Relativity with Cosmological Constant and the Accelerating Universe
}

\author{
Yves Pierseaux \\ Université Libre de Bruxelles, Brussels, Belgium \\ Email: ypiersea@ulb.ac.be
}

Received 22 July 2014; revised 18 August 2014; accepted 12 September 2014

Copyright (C) 2014 by author and Scientific Research Publishing Inc.

This work is licensed under the Creative Commons Attribution International License (CC BY). http://creativecommons.org/licenses/by/4.0/

cc) (i) Open Access

\section{Abstract}

A Minkowskian solution of the equation of General Relativity (as written by Einstein in 1915) is trivial because it simply means that both members of the equation are equal to zero. However, if alternatively, one considers the complete equation with a non-zero constant $\Lambda$ (Einstein 1917), a Minkowskian solution is no longer trivial because it amounts to impose a constraint on the right hand side of the equation (i.e. a non-null stress-energy tensor). If furthermore one identifies (as usual) this tensor to the one of a perfect fluid, one finds that this fluid has a positive energy density and a negative pressure that depend on the three constants of the equation (i.e. gravitational constant $G$, cosmological constant $\Lambda$ and velocity of light c). When doing that (§1), one has to consider the "Minkowskian Vacuum" as a physical object of GR (an enigmatic non-baryonic Minkowskian fluid). Can one build a model of this object on the basis of a dynamical equilibrium between the effective gravitational attraction due to the positive energy density versus the negative pressure repulsion? We propose to study such a model, where the (enigmatic) fluid is assumed to exist only in a limited sphere whose surface acts like a "test body" sensitive to the gravitational field created by the fluid. No static equilibrium exists, but a pseudoNewtonian "dynamical equilibrium" (\$2) can be reached if the pseudoEuclidean fluid is in state of expansion. Up to there, we have simply constructed a model of an "abstract Universe" (i.e. the limited sphere: There is no fluid outside this sphere!) that gives to a (purely mathematical) constant $\Lambda$ a concrete physical meaning. We discover finally that our expanding fluid has not only dynamical (gravitational) properties (§3) but also optical properties that are connected with Doppler Redshift (\$4). Remembering that recent observations in Cosmology indicate that the "real Universe" seems to be "Flat" and in "Accelerated Expansion"; remembering also (after all) that the archetypal Flat Universe is simply a Minkowskian Universe, we logically wonder if the unexpected Minkowskian global solution, could not be also a significant cosmological model (conclusion). 


\section{Keywords}

\section{General Relativity, Minkowskian Fluid, Cosmological Constant, Accelerating Universe}

\section{Enigmatic Minkowskian Fluid Deduced from Complete Einstein's Equation}

Let us consider Einstein's basic equation [1] of General Relativity (GR) completed by a positive mathematical constant $\Lambda>0$ [2], that has a priori nothing to do with Cosmology (with $g_{\mu v}$ Riemanian metric, $G_{\mu v}$ Einstein's curvature tensor, $T_{\mu v}$ stress-energy tensor, $G$ gravitational constant and $c$ light velocity):

$$
G_{\mu v}+\Lambda g_{\mu v}=\frac{8 \pi G}{c^{4}} T_{\mu \nu}
$$

In order to discover the physical meaning of this constant $\Lambda>0$, let us simplify with $G_{\mu v}=0$ the Equation (1) by introducing Minkowskian metric $g_{\mu v}=\eta_{\mu \nu}=\left(\begin{array}{cccc}1 & 0 & 0 & 0 \\ 0 & -1 & 0 & 0 \\ 0 & 0 & -1 & 0 \\ 0 & 0 & 0 & 1\end{array}\right)$. We obtain a tensor of Minkowskian Vacuum (2):

$$
T_{\mu v}^{\mathrm{VACUUM}}=\frac{\Lambda c^{4}}{8 \pi G} \eta_{\mu v}
$$

Let us now associate to this tensor (2) the one of a perfect relativistic fluid:

$$
T_{\mu \nu}^{\mathrm{VACUUM}} \equiv(p+\rho) \frac{u_{\mu} u_{v}}{c^{2}}-p \eta_{\mu v}
$$

Minkowskian Vacuum is then simulated by an enigmatic fluid with a positive density of energy $\rho=\frac{\Lambda c^{4}}{8 \pi G}$ and a negative pressure ${ }^{1}: \quad p=-\frac{\Lambda c^{4}}{8 \pi G}$

$$
T_{\mu \nu}^{\mathrm{VACUUM}}=T_{\mu \nu}^{\mathrm{GR}}=\left(\begin{array}{cccc}
\rho & 0 & 0 & 0 \\
0 & p & 0 & 0 \\
0 & 0 & p & 0 \\
0 & 0 & 0 & p
\end{array}\right)=\frac{\Lambda c^{4}}{8 \pi G}\left(\begin{array}{cccc}
1 & 0 & 0 & 0 \\
0 & -1 & 0 & 0 \\
0 & 0 & -1 & 0 \\
0 & 0 & 0 & 1
\end{array}\right), \quad p+\rho=0
$$

Our enigmatic Minkowskian fluid becomes a physical object in the framework of (complete) GR. Before the examination of the physical properties of our fluid determined by three basic constants ( $\Lambda, G$ and $c$ ) (§2), let us formulate two remarks.

REMARK 1 Our non-usual fluid (4) cannot be confused with usual perfect fluid (4-SR) in the framework of standard Special Relativity (SR). In this case, we have $u_{\mu} u_{v}=0$ in proper system for all components except for purely temporal components $u_{0} u_{0}=c^{2}=1$

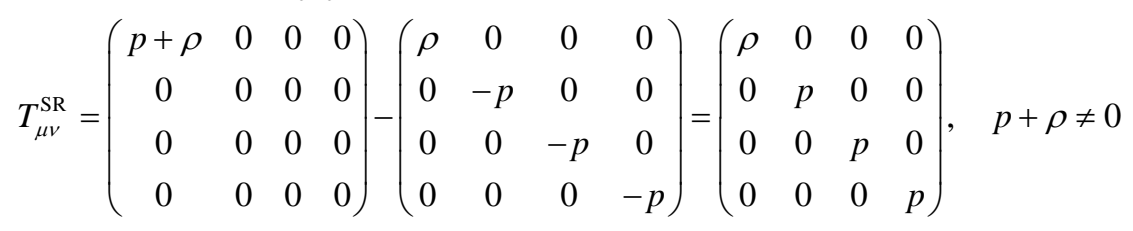

\footnotetext{
${ }^{1}$ This negative pressure of vacuum seems to be a stranger in SR. However, on the only basis of LT in 1905, Poincaré (1853-1912) introduced such a negative pressure of ether in order to define a punctual electron by its electromagnetic field [3]. He suspected the gravitational origin of his negative pressure but he did not succeed to find the exact relation between his "gravitational" scalar field $p$ and the gravitational constant $G$.
} 
Any relativistic usual perfect fluid has a positive pressure $p\left(0 \leq p \leq \frac{1}{3} w_{\mathrm{em}}\right)^{2}$. In this way our enigmatic fluid is no longer a usual fluid in "immutable Minkowskian Vacuum" (standard SR) but it is the (Classical) Minkowskian Continuum itself.

REMARK 2 Our non-usual (classical) fluid (2) cannot be confused with usual (quantum) black energy (2bis) in the framework of Cosmology. Standard method in Cosmology consists in associating a supplementary stress-tensor $T_{\mu v}^{\Lambda}$ to a cosmological constant (CC $\Lambda$ ) in the second member of (1) in order to have a second contribution to $G_{\mu \nu}:($ with $c=1)$ :

$$
G_{\mu v}=8 \pi G T_{\mu v}-\Lambda g_{\mu v}=8 \pi G\left(T_{\mu v}-T_{\mu \nu}^{\Lambda}\right) \quad \text { with } T_{\mu v}^{\Lambda}=\frac{\Lambda}{8 \pi G} g_{\mu v}=\rho_{\Lambda} g_{\mu v}
$$

(2-Riemann)

By associating $T_{\mu \nu}$ stress-energy tensor to the one of a perfect relativistic fluid $T_{\mu v}^{\Lambda}=(p+\rho) u_{\mu} u_{v}-p g_{\mu \nu}$ we usually obtain a fluid (black energy of "quantum vacuum") characterized by an unknown Riemanian metric $g_{\mu v}$ (2-Riemann) whilst in (2) the metric is determined a priori Minkowskian. In standard Cosmology, Minkowskian limit can only be a trivial result of a very improbable compensation $T_{\mu \nu}-T_{\mu \nu}^{\Lambda}=0$.

\section{Thermodynamical Properties of Minkowskian Fluid and Unstable Static Model}

Basic condition $\rho+p=0$ (4) gives a new physical interpretation of Minkowskian metric as a fluid.

$$
g_{\mu \nu}=\eta_{\mu \nu} \Leftrightarrow \rho+p=0=h
$$

The geodesic of a material point is usually determined in Minkowskian space-time as a straight line. But here we have a point of space-time continuum itself. In order to discover physical properties of our enigmatic fluid the only possible point of departure is local thermodynamical properties given by (4-bis): where $h$ is null density of enthalpy. Given that $\rho$ is a density of energy of fluid, we have by integration a finite volume $V$ with a finite energy $U$ :

$$
U+p V=0=H
$$

By differentiation we obtain:

$$
\mathrm{d} U+p \mathrm{~d} V=0=\mathrm{d} H=h
$$

that seems to trivially return to (4-bis) with reduction of element of volume $d V \neq 0 \quad(\mathrm{~d} H=h \mathrm{~d} V=0)$. Usually it is claimed that Minkowskian vacuum would be static $(\mathrm{d} V=0)$. Let us consider, at flat Minkowskian limit, an Euclidean sphere of fluid:

${ }^{2}$ For example we have free electromagnetic (em) isotropic field ( $\left.w_{\mathrm{em}}, p_{\mathrm{em}}, p_{\mathrm{em}}, p_{\mathrm{em}}\right)$ with null trace $p_{\mathrm{em}}=+\frac{1}{3} w_{\mathrm{em}}$. According to Poincaré, we have to add a special tensor to the tensor of em field in order to define Poincaré's electron: $(c=1)$ :

$T_{\mu v}^{\text {electron }}=T_{\mu v}^{\mathrm{em}}+p_{\mathrm{em}} \eta_{\mu v}=\left(p_{\mathrm{em}}+w_{\mathrm{em}}\right) u_{\mu} u_{v}=\frac{4}{3} w_{\mathrm{em}} u_{\mu} u_{v}$. In proper system, we have:

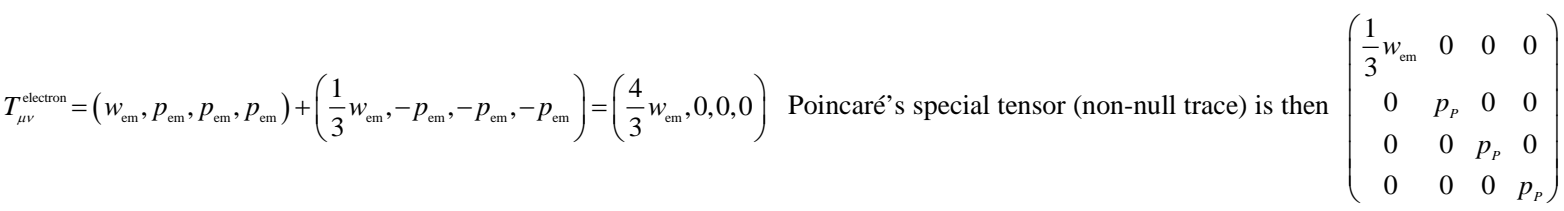
corresponding to negative Poincaré's non-em pressure $p_{\text {Poincare' }}=p_{P}=-\frac{1}{3} w_{\mathrm{em}}$. This is the reason why, one admits usually (von Laue) that $3 / 4$ of electron inertial mass $\left(\frac{4}{3} m_{\mathrm{em}}=m_{e}\right)$ comes from electromagnetic origin. We showed in "La Structure Fine de la Relativité Restreinte" that there is an enigmatic connection between Poincaré's electron (1905) and Einstein's (quantum) photon (light complex, 1905, see note 10).

${ }^{3}$ Let us note that the "vacuum" $T_{\mu v}^{\Lambda}$ in (2-Riemann) depends on $g_{\mu v}\left(G_{\mu v} \neq 0\right)$ that indicates a presence of usual matter whilst the vacuum in (2) is radically without any usual (baryonic) matter. 


$$
V=\frac{4}{3} \pi R^{3}, \quad U=\frac{4}{3} \pi \rho R^{3}
$$

At Minkowskian limit we have also to take into account Einstein’s relation of "materialization” of energy:

$$
U=M c^{2}, \quad M=\frac{4}{3} \pi \frac{\rho}{c^{2}} R^{3}
$$

How can we test the behavior (static or not static) of such a Euclidean Sphere of fluid? Let us consider a test point (infinitesimal pseudomass ${ }^{4} \mu=\mathrm{d} M$ ) on the surface of the sphere. We have to introduce the gravitational constant because $\rho_{\text {VACUUM }}=\frac{\Lambda c^{2}}{8 \pi G}$. We suggest then to study a Newtonian model where the Minkowskian fluid is assumed to exist only in a limited sphere whose surface acts like a "test body" sensitive to the gravitational field created by the fluid. The surface is submitted to gravitational attractive potential:

$$
\Phi=-\frac{G M}{R}=-\frac{4}{3} \pi G \frac{\rho}{c^{2}} R^{2}, \quad U_{P}=-\frac{G M \mu}{R}=-\frac{4}{3} \pi G \frac{\rho}{c^{2}} R^{2} \mu
$$

Then the surface of the fluid will collapse towards the center of the sphere given that we have only attractive potential energy. So a static finite sphere of our fluid is unstable $(\Delta V=V \mapsto 0)$ and the Minkowskian solution (4-bis) seems impossible. We rediscover in this way that standard immutable Minkowskian vacuum must be defined without gravitation.

\section{Dynamical Properties of Fluid, Radial Expanding Universe and Scalar Field of Gravitation}

The existence of our fluid is directly connected with Minkowskian (Pseudo-Euclidean) space-time, where basically the time is not separated from space (2). Let us thus consider that thermodynamical differential $\mathrm{d} V$ variation of volume of fluid is a temporal variation $\mathrm{d} V(t)$ :

$$
\mathrm{d} U(t)+p \mathrm{~d} V(t)=0
$$

In this way, Equation (6) is no longer trivial. We have a variable volume $V(t)$ coupled with a constant density

$$
U(t)=\rho V(t)=\frac{4}{3} \pi \rho R^{3}(t)=M(t) c^{2}
$$

Let us now consider that the Newtonian law of gravitation is also variable with a temporal gravitational potential $\Phi(t)=-\frac{G M(t)}{R(t)}$. Our test point $(\mu=\mathrm{d} M)$ at radial distance $R(t)$ has therefore a positive radial velocity $\frac{\mathrm{d} R(t)}{\mathrm{d} t}=\dot{R}(t)$. Potential energy $U_{P}=-\frac{G \mu M(t)}{R(t)}$ can be then now compensated by kinetics energy $U_{C}=\frac{1}{2} \mu \dot{R}(t)^{2}:$

$$
\frac{1}{2} \dot{R}(t)^{2}-\frac{G M(t)}{R(t)}=0 \Rightarrow \frac{1}{2} \dot{R}(t)^{2}-\frac{4}{3} \pi G \frac{\rho}{c^{2}} R(t)^{2}=0
$$

( $\mu$ disappears). This Pseudo-Newtonian model $l^{5}$ of Pseudo-Euclidean fluid is based on a dynamical equilibrium "sphere-test body" between attraction and repulsion. We obtain in this way a stability of expanding sphere with a radial enigmatic (Remark 3) “escape velocity” $\dot{R}(t)>0$ :

$$
\dot{R}(t)=\frac{R(t)}{c} \sqrt{\frac{8}{3} \pi G \rho}=c R(t) \sqrt{\frac{\Lambda}{3}}
$$

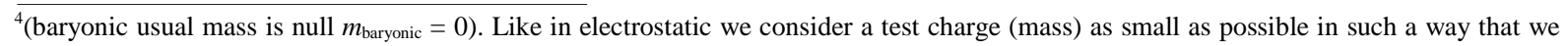
have no modification of the electrostatics field.

“"Pseudo” because Newton's law depending on time is not the usual point of view. We adopt in this way, in accordance with a GR point of view, that we can extend Newton's law of gravitation from material baryonic particles m to points of space $M$.
} 
If we suppose a finite spherical volume of fluid in dynamical equilibrium then it is in exponential expanding (11). Escape velocity (13) disappears if and only if $\Lambda=0$. Physical meaning of mathematical constant $\Lambda$ is now clarified by Minkowskian solution that implies the introduction (from 13) of a GLOBAL SCALE FACTOR $R(t)$ (with Minkowskian metric, 2 or see 19):

$$
R(t)=R_{H} \mathrm{e}^{\sqrt{\frac{\Lambda}{3} t}}
$$

with a constant of integration $R(0)=R_{H}$ that seems, at first sight, not depending on $\Lambda$.

We can also define a constant of expansion of Fluid (Vacuum) that we suggest to note $H_{\Lambda}$ (15 left):

$$
H_{\Lambda}=\frac{\dot{R}(t)}{R(t)} \quad \rho_{\mathrm{VACUUM}}=\frac{3 H_{\Lambda}^{2} c^{2}}{8 \pi G}
$$

together with a density inside the sphere (15 right). Our model supposes that there is no fluid $(\rho=0)$ outside the sphere of fluid $\left(\rho_{\text {vacuum }}\right)$. Given that the fluid simulates space-time continuum itself, there is nothing $(\rho=0)$ outside the sphere. Everything happens as if our sphere was a "Universe". By introducing mathematical constant $\Lambda$ in (1) we are thus naturally led to a theory of Universe, i.e. a cosmological interpretation:

$$
R(t)=R_{H} \mathrm{e}^{H_{\Lambda} t} \Leftrightarrow R(t)=\frac{c}{H_{\Lambda}} \mathrm{e}^{H_{\Lambda} t}
$$

Our model explains then why Hubble's expansion is necessarily a global expansion (no local observed effect of expansion). If the constant of integration $R_{H}$ (15)-(16), i.e. a global constant, is not equal to $c / H$ (if $\dot{R}(0) \neq c$ see Remark 3), there would exist two global constants of Hubble. This would be a nonsense. In order to have Pseudo-Newtonian model of Pseudo-Euclidean fluid without contradiction, we must have $H_{\Lambda} R_{H}=C(16)^{6}$.

From $\frac{\mathrm{d}}{\mathrm{d} t}\left(\frac{\dot{R}(t)}{R(t)}\right)=\frac{R(t) \ddot{R}(t)-\dot{R}(t)^{2}}{R^{2}(t)}=\dot{H}_{\Lambda}(t)=0$ we can define from radial acceleration $\ddot{R}(t)$ also a (standard) parameter of deceleration that we suggest to note $q_{\Lambda}$ :

$$
q_{\Lambda}=-\frac{R(t) \ddot{R}(t)}{\dot{R}(t)^{2}}=-1
$$

$q_{\Lambda}$ is here negative and implies thus an acceleration of expansion. Initial condition of (17) $t=0$ are:

$$
\frac{R(0) \ddot{R}(0)}{\dot{R}(0)^{2}}=\frac{R_{H} \alpha_{\Lambda}}{c^{2}}=1
$$

Initial conditions mean that $R(0)=R_{H}$ and $\ddot{R}(0)=\alpha_{\Lambda}$ define "horizon values" exactly on the same way that $\dot{R}(0)=c$ defines a "horizon value" (Remark 3 ). We obtain a basic minimal relativistic acceleration".

We deduce a pseudoNewtonian scalar field of gravitational force with a global principle of equivalence "acceleration-gravitation” $\frac{\ddot{R}(t)}{2}=G \frac{M(t)}{R(t)^{2}}$. Our dynamical Universe supposes then, with $\frac{1}{2} \dot{R}^{2}(0)-G \frac{M(0)}{R(0)}=0$,

${ }^{6}$ Our pseudo-Newtonian model, entirely based on (12), cannot be confused with historical Friedman's Newtonian model. Friedman considered a sphere of material fluid with variable radius $R(t)$ and material density $\rho(t)$ but, unlike (12), with constant mass $M$ of Universe $M=V(t) \rho(t)=\frac{4}{3} \pi R^{3}(t) \rho(t) \Rightarrow \frac{\mathrm{d} M}{\mathrm{~d} t}=\frac{\mathrm{d}}{\mathrm{d} R}\left(\rho R^{3}\right)=0$. He considered a test body at radial distance with radial velocity $\dot{R}(t)$ and therefore he obtained an equation with $E=0$ (in standard notation $K=-\frac{2 E}{m}=0$ ): $E=E_{c}+E_{p}=\frac{1}{2} m \dot{R}^{2}(t)-\frac{4}{3} \pi G m \rho(t) R^{2}(t)=0 \Rightarrow\left(\frac{\dot{R}(t)}{R(t)}\right)^{2}=\frac{8 \pi G \rho_{c}}{3}$. With critical constant density he obtained $H(t)=H$ and therefore (14). Nevertheless in Friedman's prerelativistic Newtonian model the total mass $M$ of Universe is constant whilst, in our Pseudo-Newtonian model, there is a typically relativistic variable mass of vacuum $(c=1) \quad M(t)=M_{H} \mathrm{e}^{3 H_{\Lambda} t}$ with $M(0)=M_{H}=\frac{1}{2} \frac{c^{2}}{G} R_{H}=\frac{1}{2} \frac{c^{2}}{G} \sqrt{\frac{3}{\Lambda}}$.

${ }^{7}$ We rediscover here in the framework of GR and SR, Milgrom's idea of minimal acceleration. 
an initial linear density $\frac{M(0)}{R(0)}=\frac{1}{2 G} c^{2}$ (together with an initial force $\frac{1}{2 G} c^{4}$ and power of expansion $\frac{1}{2 G} c^{5}$ ).

REMARK 3 An important objection could be formulated at this stage: Our Pseudo-Newtonian model would not be a Pseudo-Euclidean model because our basic Equation (12) uses a non-relativistic form of energy.

Everybody knows how to write kinetics energy for a material particle $m c^{2}(\gamma(\beta)-1)$. Here we do not have a material point but a point of fluid in the framework of GR. Escape velocity $\dot{R}(t)$ for such a point can be as large as we wish (not limited by c). Pseudo-Newtonian Equation (12) is a relativistic equation because velocity of light plays a basic role. In fact, the escape velocity $\dot{R}(t)$, of a point of space itself, is limited by $c$ but not in usual meaning $\beta<c$ with domain of variation $\left[0, c\left[\right.\right.$. Indeed, in order to avoid a supplementary constant $R_{H}$ (14), if we admit for the initial velocity (13) $\dot{R}(0)=c R_{H} \sqrt{\frac{\Lambda}{3}}=c$, the domain $] c, \infty[$ of variation of $\dot{R}(t)$ is limited by c. We rediscover in this way a basic tachyonic Pseudo-Euclidean "light-space-time" structure. We have to expect then optical properties of fluid.

\section{Optical Properties of Fluid of Photons and Bondi's Doppler Redshift Factor}

In Cosmology our model is very near the model of de Sitter's empty $(\rho=p=0)$ Universe. The latter is also in exponential expansion ${ }^{8} a(t)=A \mathrm{e}^{\sqrt{\frac{\Lambda}{3}} t}$ with $H_{\Lambda}=\frac{\dot{a}(t)}{a(t)}$ and acceleration $q=-1$. Lemaitre' scale factor $a(t)$ is introduced in de Sitter's metric (23) [4].

$$
\mathrm{d} s^{2}=c^{2} \mathrm{~d} t^{2}-a^{2}(t)\left(\mathrm{d} r^{2}+r^{2} \mathrm{~d} \theta^{2}+r^{2} \sin ^{2} \theta \mathrm{d} \phi^{2}\right)
$$

whilst our scale factor $R(t)$ is globally induced from (12). In de Sitter's model, the constant A(“A = 1") in $a(t)=A \mathrm{e}^{\sqrt{\frac{\Lambda}{3}} t}$ is then not a global constant determined by initial conditions of the problem (like $R_{H}$ in 16 ).

With condition of radiality $\mathrm{d} \theta=\mathrm{d} \phi=0$ we have respectively de Sitter's metric and Minkowski's metric:

$$
\mathrm{d} s^{2}=c^{2} \mathrm{~d} t^{2}-a^{2}(t) \mathrm{d} r^{2} \text { de Sitter } \mathrm{d} s^{2}=c^{2} \mathrm{~d} t^{2}-\mathrm{d} r^{2} \text { Minkowski } C C \Rightarrow R(t)
$$

that are both particular cases of non-static [5] radial Robertson-Walker's metric $(\mathrm{d} y=\mathrm{d} z=0)$

$$
\mathrm{d} s^{2}=c^{2} \mathrm{~d} t^{2}-\frac{a^{2}(t)}{1-K r^{2}} \mathrm{~d} r^{2}
$$

with local (in metric) parameter of Gaussian curvature $K=0$.

Let us now introduce the limit of light velocity with $\mathrm{ds}^{2}=0$ first in the flat metric of de Sitter $(K=0)$ :

$$
\mathrm{d} t^{2}-a^{2}(t) \mathrm{d} r^{2}=0 \quad \text { de } \operatorname{Sitter}(c=1)
$$

Usually one deduces from de Sitter model the following formula of Redshift $z=\frac{\Delta \lambda}{\lambda}$ by Doppler effect in GR

$$
1+z=\frac{a\left(t_{\text {reception }}\right)}{a\left(t_{\text {emission }}\right)}
$$

(with standard notations of the time of emission of radial photon from a remote galaxy towards the time of reception in our galaxy). Moreover with two usual cosmological measurable parameters $H$ and $q$, we obtain the following standard development into series:

$$
1+z \simeq 1+H r_{0}+\left(1+\frac{1}{2} q\right) H^{2} r_{0}^{2}+\cdots=1+\beta_{s}+\left(1+\frac{1}{2} q\right) \beta_{s}^{2}+\cdots
$$

\footnotetext{
${ }^{8}$ In the model of Einstein-de Sitter (1932) there is also a Parabolic-Euclidean solution $(K=0)$ with Critical density. Nevertheless it is a model (with $\Lambda=0$ ) for usual matter. This model is today obsolete because usual matter seems to occupy only $1 \%$ of critical density. Hoyle's Steady State is also based on metric (18-de Sitter) with a null CC. Minkowskian fluid involves a perfect cosmological principle
} 
where $\beta_{s}=H r_{0}$ is standard law of Hubble (with radial comobile distance $r_{0}$ ). Recall that $\beta_{s}=\frac{v}{c}$ (s for space) is not the velocity between two galaxies (two material $\beta_{m}$ points) but a velocity $\beta_{s}=H r_{0}$ between the "points" (elements of volume) of space itself occupied by galaxies. In de Sitter's case we have thus:

$$
1+z \simeq 1+\beta_{s}+\frac{1}{2} \beta_{s}^{2} \cdots
$$

Let us now follow the same reasoning for our spherical fluid. Optical property of our fluid is given by Minkowskian limit $\left(\mathrm{d} t^{2}-\mathrm{d} r^{2}=0\right)$ for velocity of light $c=1$ : (Minkowskianscale factor, $R(t)$, (19))

$$
1+z=\frac{R\left(t_{\text {reception }}\right)}{R\left(t_{\text {emission }}\right)}=k_{s}=\sqrt{\frac{1+\beta_{s}}{1-\beta_{s}}}
$$

in contrast with (21). Let us recall that Einstein's standard SR Doppler radial factor for material point is $k_{m}=\sqrt{\frac{1+\beta_{m}}{1-\beta_{m}}}$ ) where $z$ can be as large as we wish $\left(\beta_{s m}<1\right)^{9}$. Equation (24) involves then a "GR interpretation" (velocity of point of space) of Einstein's Doppler formula [7]. With $q=-1$ (in 25) we have precisely (23) until the second order.

$$
k_{s}=k_{\mathrm{Bondi}}=\sqrt{\frac{1+\beta_{s}}{1-\beta_{\mathrm{s}}}} \simeq 1+\beta_{\mathrm{s}}+\frac{1}{2} \beta_{\mathrm{s}}^{2}+\frac{1}{2} \beta_{\mathrm{s}}^{3}+\frac{3}{8} \beta_{\mathrm{s}}^{4} \cdots
$$

We suggest then the conjecture that the parameters of expanding universe is given by famous "Bondi's factor" [8] at any order (25). An expanding sphere of light (§3) is then inseparable from an expanding sphere of space (§4). We have thus perhaps here a new way towards CBR.

For the coherence of our model of points of space without baryonic mass " $p+\rho=0$ ”, we need for the photon a null rest mass $m=0$ in such a way that in the perfect fluid (in 3), we would have " $p+\rho=0$ ” in front of the term of four-velocity $\frac{u_{\mu} u_{v}}{c^{2}}$ for “particle” (see note 2 Poincaré's electron) $)^{10}$.

\section{Conclusion: Relativistic Effect of (Anti)Gravitational Scalar Field and Dark Energy}

We showed the existence of a simple unexpected global Minkowskian solution of Einstein's complete (with CC) equation of GR. The logical sequence from Pseudo-Euclidean solution (2) towards the Pseudo-Newtonian Fluid (12) is the following $(c=1)$ :

$$
T_{\mu \nu}^{\mathrm{VIDE}}=\frac{\Lambda}{8 \pi G} \eta_{\mu \nu} \Rightarrow p+\rho=0 \Rightarrow \mathrm{d} U(t)+p \mathrm{~d} V(t)=0 \Rightarrow \frac{1}{2} \dot{R}(t)^{2}-\frac{4}{3} \pi G \rho R(t)^{2}=0
$$

${ }^{9}$ We have therefore $\beta_{s}=\frac{v}{c} \quad v<c$ and $\beta_{s}=\frac{r_{0}}{R_{H}} \quad r_{0}<R_{H}$ as well $\left(\beta_{s}=\frac{v}{c}=H r_{0}\right)$. Everything happens as if, underlying our model, there is a new DSR (Doubly Special Relativity) a SR with a second constant $R_{H}$, i.e. a basic (maximal) length $R_{H}$ (in Minkowskian scale hyperbolas) directly connected with a minimal acceleration [6]. A possible connection between the hyperbolic velocity $\dot{R}(t)>c$ and $\beta_{\mathrm{s}}<c$ is suggested in [5].

${ }^{10}$ Minkowskian gravitational fluid can be also directly induced from equation of perfect fluid (3). Indeed if we introduce Einstein’s photon (light complex in 1905 [7]), a particle of null rest mass (unlike Poincaré's purely wavy representation of light, see note 2), we have in this case in (3) " $p+w_{\mathrm{em}}=0$ ", where $p$ cannot be the usual em pressure. In 1905, Poincaré's transformation of Energy of an electron $(v<c)$ and Einstein's transformation of Energy of a light complex (photon, $v=c$ ) are together deduced with the same Lorentz transformation of a moving sphere around a moving point. Einstein considered a moving sphere at light velocity with SPHERICAL density of PLANE wave $w_{\mathrm{em}}$ ! SIC! Lorentz (and Planck, von Laue...) considered that it was impossible in the framework of classical em. In both cases (Poincaré's electron and Einstein's photon), we must have then a strange pressure. When he discovers the (quantum) proportionality between energy and frequency of light complex, Einstein had added in optics a new quantum particle of null rest mass that supposes the unexpected existence of a strange (antigravitational) negative pressure. Lorentz was right: Einstein’s closed light sphere is impossible without the equilibrium. $p_{\text {gravitation }}=-w_{\text {em }}$. Let us note that it is a new way towards a natural "quantization" of GR (in the meaning of a black body) because classical wavy representation of light is not compatible with GR (see also usual gravitational redshift). 
Minkowskian metric (infinitesimal interval) involves (with CC) then a global scale factor $R(t)$. We wonder if we can introduce such a scale factor in a finite interval in another paper [9]. From relativistic pseudoNewtonian Equation (12), we deduce dynamical properties $H_{\Lambda}=\frac{\dot{R}(t)}{R(t)}, \quad q_{\Lambda}=-\frac{R(t) \ddot{R}(t)}{\dot{R}(t)^{2}}=-1$ and optical property (24) $1+z=\frac{R\left(t_{\text {reception }}\right)}{R\left(t_{\text {emission }}\right)}=k$ with Bondi's factor reinterpreted as a Redshift in GR .

Dynamical (§1, §2, §3) and optical (§4) properties of our Minkowskian fluid (or Continuum) are thus compatible with the most recent cosmological observations ([10]-[12]):

1) Hubble's Redshift,

2) Parameter of curvature near zero $(K=0)$,

3) Density near "critical density",

4) Parameter of acceleration near $q=-1$,

5) The Dark energy connected with a non-null CC (note 6).

Einstein's SR in 1905 consisted in dissolving a ghost: The old electromagnetic ether. Our relativistic approach involves also the dissolution of a ghost: the Dark Energy. This new cosmological ether becomes a pure relativistic effect of Minkowskian solution with CC. Unlike usual Quantum approach of Vacuum (Lemaître) our approach consists in simulating properties of Vacuum with a "Classical (apparently at the departure) Continuum". With quantum representation of light (note 10), our model becomes compatible, for example, with a continuum spectrum of a "black body" in Universal Vacuum.

\section{Acknowledgements}

I would like first to thank Jean Reignier (ULB). I thank also Laurent Favart (IIHE, ULB), Jan de Bruyne (IIHE, ULB), Nicolas Vansteenkiste (ESI-heb), Frédéric Servais (ESI-heb) and Eytan Levy (ESI-heb).

\section{References}

[1] Einstein, A. (1916) Annalen der Physik, 354, 769-822.

[2] Einstein, A. (1917) Kosmologische Betrachtungen zur Allgemeinen Relativitätstheorie. In: Sitzungsberichte der Königlich Preussischen Akademie der Wissenschaften, VI, Berlin, 142-152.

[3] Poincaré, H. (1906) Rendicontidel Circolo Matematico di Palermo, 21, 129-175.

[4] de Sitter, W. (1917) Royal Astronomical Society Monthly Notices, LXXVIII, 3-28. http://dx.doi.org/10.1093/mnras/78.1.3

[5] Pierseaux, Y. (2010) From Unexpected Minkowskian Solution of Einstein’s Equation of General Relativity with Cosmological Constant to the Accelerating Universe. Revue IIHE. http://arxiv.org/abs/1009.1375

[6] Rindler, W. (2001) Relativity. Special, General and Cosmological. Oxford University Press, Oxford.

[7] Einstein, A. (1905) Annalen der Physik, 17, 891-921.

[8] Bondi, H. (1962) Relativity and Common Sense. A New Approach to Einstein. Dover Publications Inc., New York.

[9] Pierseaux, Y. (2013) Annales de la Fondation Louis de Broglie, 38, 41-55.

[10] Riess, A., et al. (1998) Astronomical Journal, 116, 1009-1038. http://dx.doi.org/10.1086/300499

[11] Peebles, P.J.E. (2002) The Cosmological Constant and Dark Energy. http://arxiv.org/abs/astro-ph/0207347

[12] Turner, M. (2002) The New Cosmology. World Scientific, Singapore. 
Scientific Research Publishing (SCIRP) is one of the largest Open Access journal publishers. It is currently publishing more than 200 open access, online, peer-reviewed journals covering a wide range of academic disciplines. SCIRP serves the worldwide academic communities and contributes to the progress and application of science with its publication.

Other selected journals from SCIRP are listed as below. Submit your manuscript to us via either submit@scirp.org or Online Submission Portal.
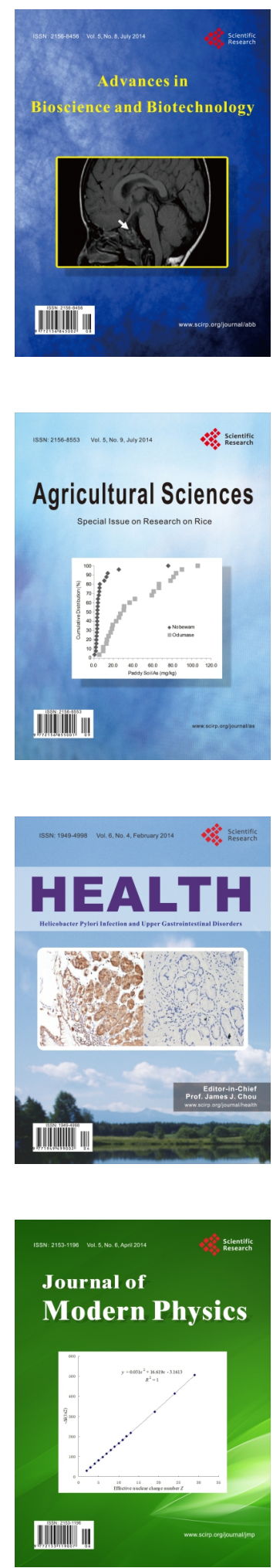
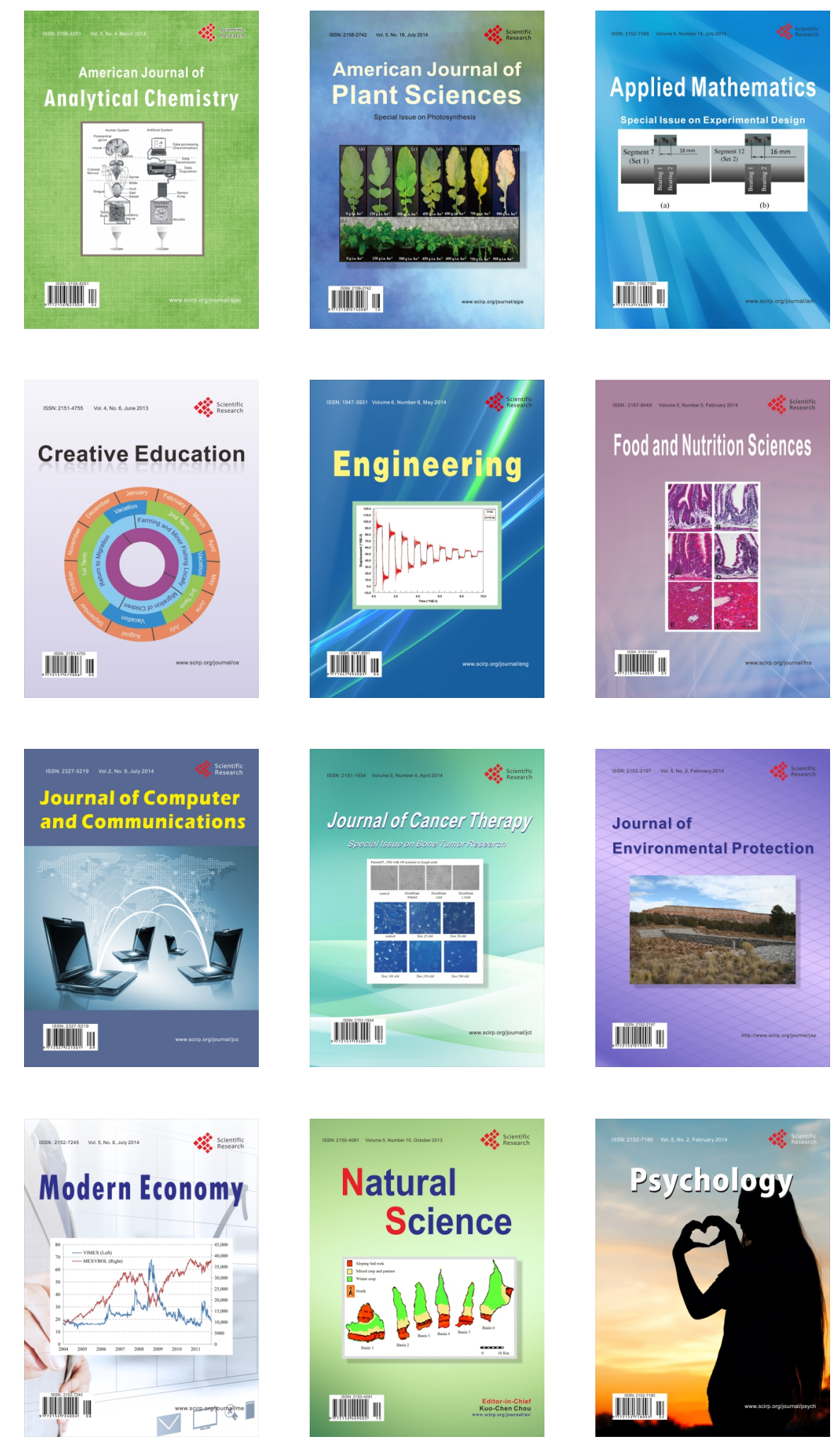